\title{
Intrinsic Fano Interference of Localized Plasmons in Pd Nanoparticles
}

\author{
Tavakol Pakizeh,* Christoph Langhammer, Igor Zorić, Peter Apell, \\ and Mikael Käll
}

\author{
Department of Applied Physics, Chalmers University of Technology, \\ Göteborg 41296, Sweden
}

Received December 16, 2008; Revised Manuscript Received January 14, 2009

\begin{abstract}
Palladium (Pd) nanoparticles exhibit broad optical resonances that have been assigned to so-called localized surface plasmons (LSPs). The resonance's energy varies with particle shape in a similar fashion as is well known for LSPs in gold and silver nanoparticles, but the lineshape is always anomalously asymmetric. We here show that this effect is due to an intrinsic Fano interference caused by the coupling between the plasmon response and a structureless background originating from interband transitions. The conclusions are supported by experimental and numerical simulation data of Pd particles of different shape and phenomenologically analyzed in terms of the point dipole polarizability of spheroids. The latter analysis indicates that the degree of Fano asymmetry is simply linearly proportional to the imaginary part of the interband contribution to the metal dielectric function.
\end{abstract}

The recent decade has witnessed an enormous research effort directed at understanding and utilizing the optical properties of nanoscale metal structures, including colloidal particles of various shapes and composition and elaborate structures manufactured through advanced lithography techniques. The key phenomenon causing this huge interest is surface plasmon resonances (SPRs), that is, free-electron density oscillations that are confined to the interface between the metal and the surrounding dielectric and that under certain circumstances can be excited resonantly by an incident electromagnetic wave. ${ }^{1}$ The SPRs generate very strong local fields at the interface, fields that can be tuned spatially and spectrally by controlling the morphology and composition of the metal nanostructure. The enhanced local fields can in turn be used to, for example, greatly amplify molecular optical cross-sections (as in surface-enhanced Raman scattering), determine the response of the SPR to a change in interfacial refractive index (which is the basis for SPR biosensors), and be made to propagate and channel information within novel nanophotonics devices. ${ }^{2-6}$ Through the application of advanced analytical techniques coupled to electrodynamics simulations, a good understanding of elementary plasmonic resonances is now at hand. However, many of the most spectacular applications in the field rely on complicated coherent interactions between elementary plasmons or between plasmons and other kinds of excitations. One example is metallic metamaterials, in which hybridiza-

* To whom correspondence should be addressed. E-mail: Tavakol.pakizeh@ chalmers.se. tion between plasmons can induce a magnetic response that in turn may lead to a negative refractive index.,3,7-9

A particularly interesting case of coherent interaction occurs if one of the elementary excitations is much broader than the other, approaching a continuum. The interaction is then known as Fano interference and it is revealed through the narrow excitation acquiring a distinct asymmetric lineshape. ${ }^{10}$ Although Fano interferences are ubiquitous in physics, with examples ranging from atomic resonance spectroscopy to phonons in superconductors, and are well known for the case of periodically structured metal films, there are few examples of the phenomenon in conjunction to nanoplasmonic systems. ${ }^{11-14}$ It has been reported that interband electronic transitions ${ }^{15}$ and nonlocal effects ${ }^{16}$ in metal nanoparticles influence the line-shape of the LSP resonances. In this letter, we show that Fano-type interference plays a crucial role in determining the spectral shape of plasmon resonances in palladium (Pd) nanoparticles. In contrast to previous nanoplasmonic examples of Fano interference, which all involve coupling between spatially localized modes (e.g., disk and ring plasmons ${ }^{11}$ or particle plasmons and molecular resonances ${ }^{12}$ ), the Pd nanoparticles exhibit an intrinsic Fano interference due to coupling between the free- and bound-electron response of the metal. As such, it can be expected to be of general importance for interpreting optical spectra of nanoparticles composed of metals that have so far received little attention in the plasmonics community.

Figure 1a displays representative experimental optical spectra of Pd nanodisks with different diameters $(D=51$, $76,110 \mathrm{~nm})$ but constant height $(h=20 \mathrm{~nm})$. The data is 


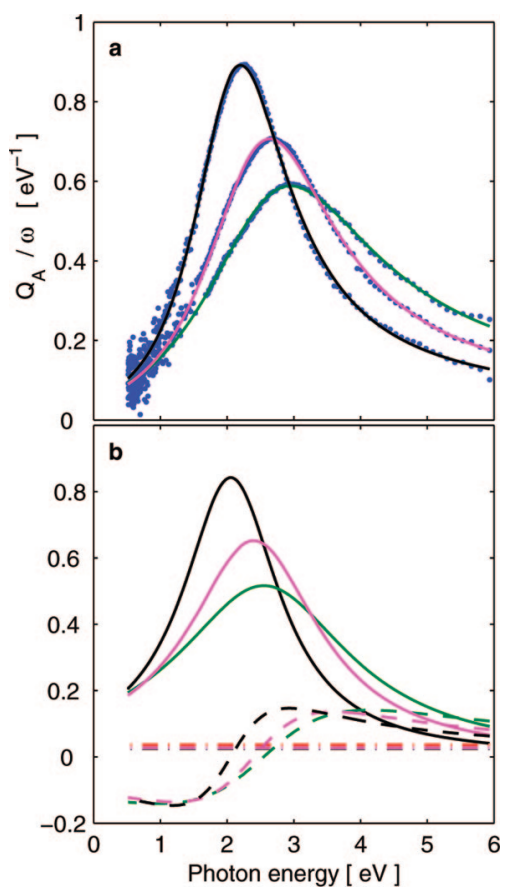

Figure 1. The measured optical response of Pd nanodisks. (a) Dotted curves show $Q_{\mathrm{A}} / \omega$ vs photon energy, where $Q_{\mathrm{A}}$ is the experimental optical absorption efficiency spectrum, for Pd nanodisks with varying diameters $D$ but constant height $h=20 \mathrm{~nm}$. The experimental data points have been fitted with the Fano function, eq 1 , according to $D=51 \mathrm{~nm}$ (green solid curve), $D=$ $76 \mathrm{~nm}$ (violet) and $D=110 \mathrm{~nm}$ (black). (b) Decomposition of the Fano functions obtained from fits to the experimental data in (a). Full lines correspond to the symmetric (Lorentzian) term in eq 1, dashed lines to the interference term, and dash-dotted lines to the constant background term. The asymmetry parameters obtained from the fits were $q=3.95(D=51 \mathrm{~nm}), q=4.9(D=76 \mathrm{~nm})$, and $q=6(D=110 \mathrm{~nm})$.

adapted from ref 17 and refers to samples prepared by hole mask colloidal lithography, ${ }^{18}$ which results in particles with edge-to-edge distances of the order $D$ or larger with no longrange order. The random arrangement of the nanodisks suppresses far-field coupling while a sufficiently large interparticle separation makes near-field coupling negligible. As reported in ref 18, the fabrication method used results in polydisperse nanodisk diameters. However, based on the measured particle size distributions ${ }^{20}$ for the three nanodisk sizes reported in this work, we estimate heterogeneous broadening contribution to the measured absorption spectra to be less than $5 \%$ of the total line-width. Consequently, the spectra are good representations of individual particle optical properties. ${ }^{19,20}$ The quantity displayed in Figure 1a is $Q_{\mathrm{A}}(\omega) /$ $\omega$, where $Q_{\mathrm{A}}(\omega)$ is the frequency dependent absorption efficiency and is obtained by dividing the absorption crosssection $\sigma_{\mathrm{A}}(\omega)$ with the geometrical area of the disk. As described in refs 17 and 20, $\sigma_{\mathrm{A}}(\omega)$ can be obtained experimentally by subtracting the scattering spectrum $\sigma_{\mathrm{S}}(\omega)$, obtained from an integrating sphere measurement, from the ordinary extinction $\sigma_{\mathrm{E}}(\omega)$ recorded in transmission mode, that is, $\sigma_{\mathrm{A}}=\sigma_{\mathrm{E}}-\sigma_{\mathrm{S}}$. The reason for plotting $Q_{\mathrm{A}}(\omega) / \omega$ is that this is linearly proportional to the imaginary part of the particle dipole polarizability, that is, $Q_{\mathrm{A}}(\omega) / \omega \propto \operatorname{Im}[\alpha(\omega)]$, which can be regarded as the most fundamental optical response function of particles in the Rayleigh limit.

Figure 2 displays theoretical spectra of nanodisks with similar dimensions as in the experiments obtained using the dispersive finite-difference time-domain (D-FDTD) method, which numerically solves Maxwell's equations in confined geometries. ${ }^{9,21}$ Using a Drude-Lorentz permittivity model to fit the experimental dielectric function $\varepsilon(\omega)=\varepsilon^{\prime}(\omega)+$ $\mathrm{i} \varepsilon^{\prime \prime}(\omega)$ of $\mathrm{Pd},{ }^{22}$ we can calculate the electromagnetic fields inside the nanodisks and obtain the absorption spectrum from $\sigma_{\mathrm{A}}(\omega) \propto \int_{V} \varepsilon^{\prime \prime}(\omega) \mathbf{E}_{\mathrm{L}}(\mathbf{r}, \omega) \cdot \mathbf{E}_{\mathrm{L}}^{*}(\mathbf{r}, \omega) \partial^{3} r$, where $\mathbf{E}_{\mathrm{L}}(\mathbf{r}, \omega)$ is the self-consistently determined local electric field and the integration runs over the volume of the particle. ${ }^{23}$ The D-FDTD simulations are clearly in excellent agreement with the experimental data. In particular, both sets of curves exhibit the same distinct asymmetry with pronounced intensity depletion at the low-energy sides of the peak maxima and a corresponding pile-up of intensity at the high-energy sides.

To characterize the spectral asymmetry seen in Figures 1 and 2, we fitted the data to the Fano line-shape function

$$
\begin{aligned}
F(\omega) & =\mathrm{A} \frac{\left(\mathrm{q}+\frac{\omega-\omega_{0}}{\Gamma}\right)^{2}}{1+\left(\frac{\omega-\omega_{0}}{\Gamma}\right)^{2}} \\
& =A\left[\frac{\left(q^{2}-1\right) \Gamma^{2}}{\left(\omega-\omega_{0}\right)^{2}+\Gamma^{2}}+1+\frac{2 q \Gamma\left(\omega-\omega_{0}\right)}{\left(\omega-\omega_{0}\right)^{2}+\Gamma^{2}}\right]
\end{aligned}
$$

Here, $q$ is inversely proportional to the degree of lineshape asymmetry, $\omega_{0}$ and $\Gamma$ are the effective resonance position and line-width, respectively, and $A$ is a constant. ${ }^{10}$ Depending on the sign of $q$, eq 1 describes a line-shape with depleted intensity on the low $(q>0)$ or high $(q<0)$ energy side of the peak. The Pd nanodisk resonances are all characterized by a positive $q$ in the range $q \in[1,7]$. We take the extremely high quality of the fits over the extended energy range from $\sim 0.7-5 \mathrm{eV}$ as evidence that the spectral asymmetry seen in Figure 1 indeed constitute a case of Fano interference.

Equation 1 is an approximation, suitable for curve-fitting purposes, of the full response function of a discrete resonance that couples to a continuum. Because of this, it is not meaningful to attempt to draw quantitative conclusions from the absolute values of the $q, \omega_{0}$, and $\Gamma$ parameters. Nevertheless, the importance of the interference phenomenon can be illustrated by separating the Fano function into its three constituent parts (see eq 1), that is, a symmetric Lorentzian, which is dominated by the discrete resonance, a constant term due to the frequency independent continuum, and an interference term, which vanishes if the coupling between the discrete resonance and the continuum vanishes. In the lower panels of Figures 1 and 2, we display the three components of the fitted Fano functions. It is clear that the interference terms give large contributions to the overall lineshapes with amplitudes of the order $30-50 \%$ of the Lorentzian contributions.

In order to elucidate the origin of the Fano interference, we need to investigate a physical model of the measured response function, that is, the particle polarizability $\alpha(\omega)$. 


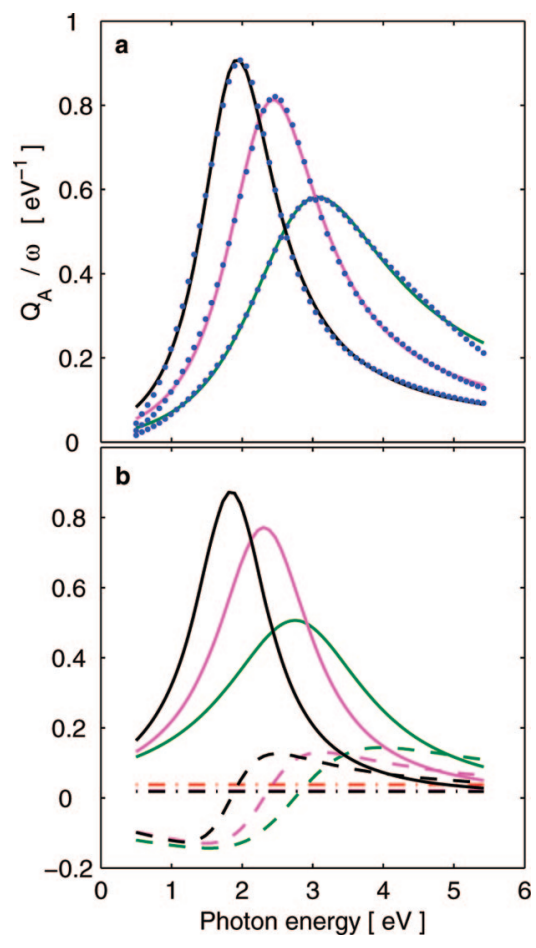

Figure 2. The calculated optical response of Pd nanodisks. Same quantities as in Figure 1, but spectra obtained from D-FDTD simulations. The nanoparticles were approximated as disks, that is, truncated cylinders with diameters $D=52 \mathrm{~nm}$ (green solid curve), $80 \mathrm{~nm}$ (violet), and $120 \mathrm{~nm}$ (black) and constant height $h$ $=20 \mathrm{~nm}$. An average surrounding refractive index $n=1.26$ was used to approximately account for the glass substrate $(n=1.52)$ and immersion medium (air) used in the experiment. The asymmetry parameters obtained from the fits were $q=3.8(D=52 \mathrm{~nm}), q=$ $6.1(D=80 \mathrm{~nm})$, and $q=7(D=120 \mathrm{~nm})$.

We first consider the polarizability of a spherical particle in vacuum taken in the Rayleigh limit, as given by the ClausiusMossotti formula. ${ }^{24}$ As is the case with most transition metals, the dielectric function of Pd can be considered to be composed of two distinct contributions, $\varepsilon=\tilde{\varepsilon}_{\mathrm{D}}+\varepsilon_{\mathrm{I}}$, where the Drude term $\tilde{\varepsilon}_{\mathrm{D}}=-\omega_{\mathrm{p}}^{2} / \omega(\omega+\mathrm{i} \gamma)$ describes the freeelectron contribution and $\varepsilon_{\mathrm{I}}$ signifies a background contribution dominated by the interband, or bound electron, response. In the absence of an interband contribution, i.e. $\varepsilon_{\mathrm{I}}=1$, insertion into the Clausius-Mossotti polarizability function yields:

$$
\begin{aligned}
\operatorname{Im}[\alpha(\omega)] \propto \operatorname{Im}\left[\frac{\varepsilon(\omega)-1}{\varepsilon(\omega)+2}\right] & =\operatorname{Im}\left[\frac{\omega_{\mathrm{LSP}}^{2}}{\omega_{\mathrm{LSP}}^{2}-\omega^{2}-\mathrm{i} \omega \gamma}\right] \\
& \approx \frac{\omega_{\mathrm{LSP}} \gamma / 4}{\left(\omega-\omega_{\mathrm{LSP}}\right)^{2}+(\gamma / 2)^{2}}
\end{aligned}
$$

where $\omega_{\mathrm{LSP}}=\omega_{\mathrm{p}} /(3)^{1 / 2}$ is the well-known localized surface plasmon (LSP) resonance of a sphere. The Lorentzian approximation holds near resonance and thus coincides (apart from prefactors) with the Fano function taken in the large- $q$ limit. The dipolar resonance of a "Drude sphere" is thus, as expected, fully symmetric. We now go one step further and incorporate a complex interband contribution $\varepsilon_{\mathrm{I}}=\varepsilon_{\mathrm{I}}^{\prime}+\mathrm{i} \varepsilon_{\mathrm{I}}^{\prime \prime}$ and a dielectric constant $\varepsilon_{\mathrm{m}}$ that characterizes the medium surrounding the nanoparticle. Further, in order to simulate the shape of the Pd nanodisks, we utilize a polarizability function describing an oblate spheroid. One then obtains

$$
\begin{aligned}
\alpha(\omega) & =V \frac{\varepsilon-\varepsilon_{\mathrm{m}}}{3 \varepsilon_{\mathrm{m}}+3 L\left(\varepsilon-\varepsilon_{\mathrm{m}}\right)} \\
& =\left(\frac{V}{3 L}\right)\left(\frac{L \omega_{\mathrm{p}}^{2}-L\left(\varepsilon_{\mathrm{I}}-\varepsilon_{\mathrm{m}}\right)\left(\omega^{2}+\mathrm{i} \gamma \omega\right)}{L \omega_{\mathrm{p}}^{2}-\left[(1-L) \varepsilon_{\mathrm{m}}+L \varepsilon_{\mathrm{I}}\right]\left(\omega^{2}+\mathrm{i} \gamma \omega\right)}\right)
\end{aligned}
$$

Note that eq 3 describes one of the polarizability components of a spheroid. In particular, it can be used to simulate the polarizability along the long axis of a nanodisk, which is what is probed in Figures 1 and 2. In this case $V=\pi D^{2} h /$ 2 , where $D$ is the diameter and $h$ is the height, and the factor $L$ is determined by the aspect ratio $D / h$ of the disk. ${ }^{24}$ What is remarkable is that this seemingly complicated function can be separated into three distinct contributions that bear a striking resemblance to the three components building up the classical Fano formula $F(\omega)$. We thus find

$$
\begin{gathered}
\operatorname{Im}[\alpha(\omega)]=\frac{V}{3 L} \operatorname{Im}\left[\hat{\alpha}_{\mathrm{L}}+\hat{\alpha}_{\mathrm{I}}-\hat{\alpha}_{\mathrm{L}} \hat{\alpha}_{\mathrm{I}}\right] \\
\hat{\alpha}_{\mathrm{L}}(\omega)=\frac{\omega_{\mathrm{LSP}}^{2}}{\omega_{\mathrm{LSP}}^{2}-\omega^{2}-\mathrm{i} \gamma \omega} ; \omega_{\mathrm{LSP}}^{2}=\omega_{\mathrm{p}}^{2} / B ; \hat{\alpha}_{\mathrm{I}}=\left(\varepsilon_{\mathrm{I}}-\varepsilon_{\mathrm{m}}\right) / B ; \\
B=(1 / L-1) \varepsilon_{\mathrm{m}}+\varepsilon_{\mathrm{I}}
\end{gathered}
$$

The component $\hat{\alpha}_{I}$ obviously contributes a pure interband term to the particle polarizability. In particular, if the interband part of the metal dielectric function can be assumed to be a complex constant, $\varepsilon_{\mathrm{I}}$, within a limited frequency interval, then this term will add a constant background ( $V /$ $3 L) \operatorname{Im}\left[\hat{\alpha}_{I}\right]$ to the particle spectrum, in analogy with the second term in eq 1 . The term $\hat{\alpha}_{L}$, on the other hand, represents the free-electron, or plasmonic, response of the particle. This term will thus contribute a Lorentzian peak to the spectrum, similar to eq 2. However, if the interband dielectric constant is complex, then the resonance frequency $\omega_{\text {LSP }}$ will also be complex. In particular, the imaginary part of $\omega_{\text {LSP }}$, which is determined by $\varepsilon_{\mathrm{I}}^{\prime \prime}$, will broaden the resonance, resulting in an effective line-width $\Gamma>\gamma / 2$. Likewise, $\varepsilon_{\mathrm{I}}$ will induce a new effective resonance position $\omega_{0} \approx \operatorname{Re}\left[\omega_{\text {LSP }}\right]$ that is down shifted compared to the pure free-electron case. The spectral contribution from the crossterm $-\hat{\alpha}_{L} \hat{\alpha}_{I}$, finally, is approximately given by $\operatorname{Im}\left[\hat{\alpha}_{I}\right] \omega_{0}(\omega$ $\left.-\omega_{0}\right) /\left[\left(\omega-\omega_{0}\right)^{2}+\Gamma^{2}\right]$, which clearly has the same functional form as the interference term in the Fano formula.

In order to substantiate the preceding arguments, we now insert a model dielectric function $\varepsilon=\tilde{\varepsilon}_{\mathrm{D}}+\varepsilon_{\mathrm{I}}$ that approximately characterizes Pd into eqs 3 and 4 . The idea is to demonstrate that the simple dipole polarizability model with an included constant interband contribution indeed gives rise to a Fano asymmetry in line with the experimental data and electrodynamics simulations in Figure 1-2. In Figure $3 \mathrm{a}$, we first plot the experimentally determined dielectric function of Pd from ref 22. We then perform a global fit to the real and imaginary parts of $\varepsilon(\omega)$ using the aforementioned model function. The best fit is found for Drude parameters 


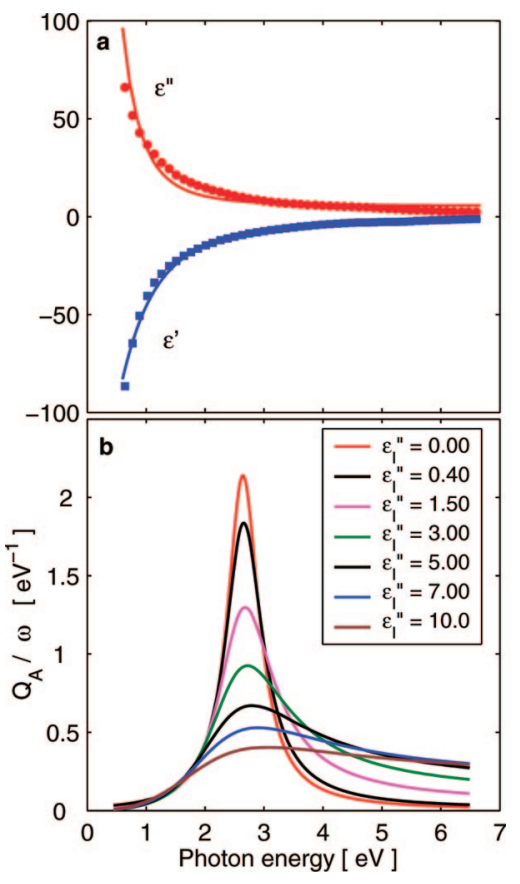

Figure 3. The Fano resonance versus imaginary component of the interband contribution. (a) Experimental dielectric data $\varepsilon(\omega)=\varepsilon^{\prime}(\omega)$ $+\mathrm{i} \varepsilon^{\prime \prime}(\omega)$ (points) of Pd from ref 22 fitted by a model function $\varepsilon=$ $\tilde{\varepsilon}_{\mathrm{D}}+\varepsilon_{\mathrm{I}}$ (solid lines). (b) Plots of $Q_{\mathrm{A}} / \omega$ using the polarizability of an oblate spheroid according to eqs 3 and 4 and the model dielectric function $\varepsilon=\tilde{\varepsilon}_{\mathrm{D}}+\varepsilon_{\mathrm{I}}$ with parameters from panel a, but with a varying imaginary part of the interband contribution $\varepsilon_{\mathrm{I}}$. The spheroid has diameter $D=80 \mathrm{~nm}$ and height $h=20 \mathrm{~nm}(L \approx 0.148)$ and is embedded in a medium with dielectric constant $\varepsilon_{\mathrm{m}}=1.26$. $^{2}$

$\omega_{\mathrm{P}} \approx 8.11 \mathrm{eV}, \gamma \approx 0.67 \mathrm{eV}$, and interband contribution $\varepsilon_{\mathrm{I}}$ $\approx 0.12+5.6 \mathrm{i}$, in fair agreement with earlier work on thin Pd films. ${ }^{25}$ Although the real part $\varepsilon^{\prime}(\omega)$ is well accounted for by a pure Drude contribution, the imaginary part $\varepsilon^{\prime \prime}(\omega)$ clearly includes a large and approximately uniform background that dominates within the energy range considered. As detailed in refs 26 and 27, this background originates in transitions between occupied and unoccupied d-like states and between the d-band and empty p-like states. In particular, the interband contribution extends far below the visible wavelength range because the Fermi level crosses the d-band manifold. ${ }^{28-30}$ This behavior is more evident for Pd than the well-known "plasmonic" metals $\mathrm{Au}$ and $\mathrm{Ag}$, which exhibit distinct interband thresholds at $\sim 2.4$ and $\sim 4 \mathrm{eV}$, respectively. ${ }^{31}$ In the case of Pd, the d-electrons overlap the s- and p-electrons in the whole range of interest, leading to an approximately uniform background. This means that the Fano effect is more clear-cut than in the case of Au for which the plasmon interact with a structured interband background around $2.4 \mathrm{eV}$. For $\mathrm{Au}$ nanospheres, the latter effect also lead to an asymmetric line-shape (not considered here), which more or less disappears if the plasmon is pushed toward lower energies for higher aspect ratio particles.

In Figure 3b, we display simulated spectra according to eqs 3 and 4 for an oblate spheroid of similar dimensions ( $L$ $\approx 0.148$ ) as in the experiments and with the same averaged dielectric surrounding. We keep all parameters in the model dielectric function constant, as given by the fit in Figure 3a,

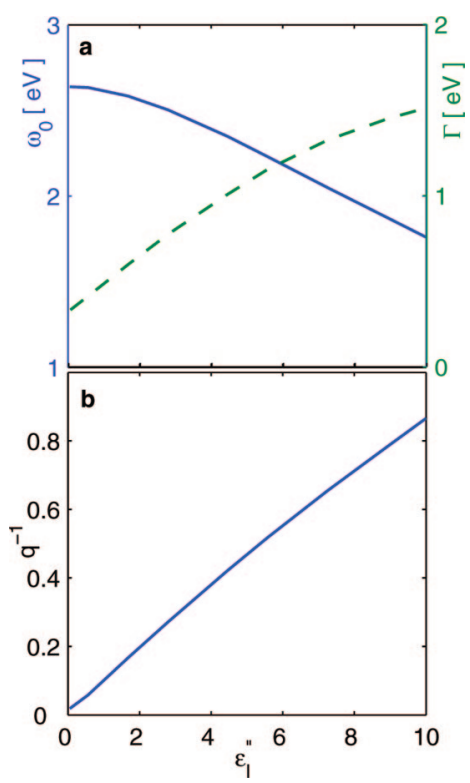

Figure 4. The Fano parameters versus imaginary component of the interband contribution. (a) Effective resonance position $\omega_{0}$ (solid line) and line-width $\Gamma$ (dashed line) (b) Asymmetry, quantified as $q^{-1}$, versus imaginary component of the interband contribution $\varepsilon_{\mathrm{I}}$. The parameters were obtained by fitting the Fano function, eq 1, to the data displayed in Figure $3 b$.

except for the imaginary part of the interband contribution, which is varied between $\varepsilon_{\mathrm{I}}^{\prime \prime}=0$ and $\varepsilon_{\mathrm{I}}^{\prime \prime}=10$. It is clear that this variation has an enormous impact on the plasmon lineshape, inducing a substantial asymmetry and broadening already at small $\varepsilon_{\mathrm{I}}^{\prime \prime}$ values. In particular, we see that the spectrum obtained for $\varepsilon_{\mathrm{I}}^{\prime \prime}$ close to the value found in the fit to the experimental dielectric function has an overall shape that is very similar to the spectra in Figures 1 and 2. We now fit the calculated spectra, shown in Figure $3 b$, to the Fano function $F(\omega)$ in order to quantify how the interband contribution $\varepsilon_{\mathrm{I}}^{\prime \prime}$ affects the line-shape parameters $\omega_{0}, \Gamma$, and $q$. The results are displayed in Figure 4 and show that the effective resonance position red-shifts continuously while the line-width increases with an increase in $\varepsilon_{\mathrm{I}}^{\prime \prime}$. The former effect is analogous to the well-known LSP shift induced by an increase in the surrounding dielectric constant $\varepsilon_{\mathrm{m}}$ or in the real part of $\varepsilon_{\mathrm{I}}$, while the latter can be interpreted as an increased decay rate of plasmons to the electron-hole pair excitations that form the interband continuum. However, the most interesting effect is seen for the asymmetry parameter $q$, which turns out to scale as $q^{-1} \approx \varepsilon_{\mathrm{I}}^{\prime \prime} / \operatorname{Re}[B]$. The degree of asymmetry is thus directly proportional to the imaginary part of the interband contribution. This effect can be traced back to the interference term in eq 4 , which is linearly proportional to $\hat{\alpha}_{\mathrm{I}}$. Accordingly, the effective line-width can be approximated as $\Gamma \approx \gamma / 2+\omega_{0} \varepsilon_{\mathrm{I}}^{\prime \prime} /(2 \operatorname{Re}[B])$, which is indeed proportional to the imaginary part of the interband contribution, shown in Figure 4a.

In summary, we have investigated optical absorption spectra of palladium nanoparticles using experimental data and electrodynamical simulations. The spectra are dominated by a highly asymmetric localized surface plasmon peak that can be fitted extremely well by the classical Fano function, 
which describes the interaction between a resonance and a continuum. Using a simple dipole polarizability approach, we show that the asymmetry arises naturally due to the presence of an interband continuum $\varepsilon_{\mathrm{I}}$ that overlaps with the plasmon resonance. Specifically, we find that the degree of asymmetry scales linearly with the strength of the imaginary part of $\varepsilon_{\mathrm{I}}$. The analysis that leads up to this conclusion is of course based on several approximations and simplifications. For example, the point dipole approximation generally needs to be adjusted for retardation and dynamic depolarization, but such finite size effects would complicate the effective polarizability function to the extent that an analysis along the lines of eq 4 becomes impossible. Furthermore, we neglect all possible multipolar contributions to the optical absorption. Both these effects are, however, incorporated into the D-FDTD simulations, which show a striking resemblance to the simple dipole spectra and to the experimental data. A more serious simplification might be that we take the interband contribution to be frequency independent. Nevertheless, the strength of the asymmetry effect seen in Figures 3 and 4 indicates that a frequency dependent $\varepsilon_{\mathrm{I}}$, compatible with the Kramers-Kronig causality relations, would not change the overall conclusion, namely that the highly asymmetric shapes of the Pd nanoparticle absorption spectra are due to an intrinsic Fano interference between localized plasmon resonances and an interband continuum. A more fundamental analysis of this phenomenon, perhaps including quantized plasmon and electron-hole pair excitations coupled along the lines of Fano's original treatment of atomic absorption lines, would be clearly valuable but goes beyond the scope of this work. We should note here that Fano interference between bulk plasmons and interband transition in Si has been observed by inelastic X-ray scattering and successfully interpreted using a jellium model.$^{33}$ Finally, we should address the generality of the above conclusions. We have here focused entirely on the case of Pd nanoparticles, which are technologically important in relation to, for example, hydrogen storage ${ }^{34}$ However, many fcc, hcp and bcc transition metals exhibit the same basic dielectric response as Pd, i.e. a free electron "Drude contribution" that overlaps with an extended interband continuum due to transitions within sp, $d$ and hybridized bands cut by the Fermi energy. One such case is $\mathrm{Pt}^{20}$ for which we have confirmed the presence of a strong Fano asymmetry for nanodisks of similar size and shape as discussed here.

Acknowledgment. This work was supported by the Swedish Foundation for Strategic Research and the Swedish Research Council.

\section{References}

(1) Kreibig, U.; Vollmer, M. Optical properties of metal clusters; Springer: Berlin, 1995.

(2) Valentine, J.; Zhang, S.; Zentgraf, T.; Avila, E. U.; Genov, D. A.; Bartal, G.; Zhang, X. Nature 2008, 455, 376-380.

(3) Cai, W. S.; Chettiar, U. K.; Kildishev, A. V.; Shalaev, V. M. Nat. Photonics 2007, 1, 224-227.

(4) Maier, S. A.; Kik, P. G.; Atwater, H. A.; Meltzer, S.; Harel, E.; Koel, B. E.; Requicha, A. A. G. Nat. Mater. 2003, 2, 229-232.

(5) Lal, S.; Link, S.; Halas, N. L. Nat. Photonics 2007, 1, 641-648.

(6) Larsson, E. M.; Alegret, J.; Käll, M.; Sutherland, D. S. Nano Lett. 2007, 7, 1256-1263.

(7) Soukoulis, C. M.; Linden, S.; Wegner, M. Science 2007, 315, 47-49.

(8) Pakizeh, T.; Abrishamian, M. S.; Granpayeh, N.; Dmitriev, A.; Käll, M. Opt. Express 2006, 14, 8240-8246.

(9) Pakizeh, T.; Dmitriev, A.; Abrishamian, M. S.; Granpayeh, N.; Käll, M. J. Opt. Soc. Am. B 2008, 25, 659-667.

(10) Fano, U. Phys. Rev. 1961, 124, 1866-1878.

(11) Hao, F.; Sonnefraud, Y.; Dorpe, P. V.; Maier, S. A.; Halas, N. J.; Nordlander, P. Nano Lett. 2008, 8, 3983-3988.

(12) Neuhauser, D.; Lopata, K. J. Chem. Phys. 2007, 127, 154715.

(13) Tribelsky, M. I.; Flach, S.; Miroshnichenko, A. E.; Gorbach, A. V.; Kivshar, Y. S. Phys. Rev. Lett. 2008, 100, 043903.

(14) Zhang, W.; Govorov, A. O.; Bryant, G. W. Phys. Rev. Lett. 2006, 97, 146804.

(15) Pinchuk, A.; Plessen, G. V.; Kreibig, U. J. Phys. D: Appl. Phys. 2004, 37, 3133-3139.

(16) Garcia de Abajo, F. J. J. Chem. Phys. C 2008, 112, 17983-17987.

(17) Langhammer, C.; Kasemo, B.; Zoric, I. J. Chem. Phys. 2007, 126, 194702.

(18) Fredriksson, H.; Alaverdyan, Y.; Dmitriev, A.; Langhammer, C.; Sutherland, D. S.; Zäch, M.; Kasemo, B. Adv. Mater. 2007, 19, 42974302.

(19) Hanarp, P.; Käll, M.; Sutherland, D. S. J. Phys. Chem. B 2003, 107, $5768-5772$

(20) Langhammer, C.; Yuan, Z.; Zoric, I.; Kasemo, B. Nano Lett. 2006, 6, 833-838.

(21) Taflove, C.; Hagness, S. C. Computational electrodynamics: The finitedifference time-domain method; Artech House: Boston, 2005.

(22) Johnson, P. B.; Christy, R. W. Phys. Rev. B 1974, 9, 5056-5070.

(23) Sun, W.; Loeb, N. G.; Fu, Q. Appl. Opt. 2002, 41, 5728-5743.

(24) Bohren, C. F.; Huffman, D. R. Absorption and scattering of light by small particles; John Wiley \& Sons: New York, 1983.

(25) Vargas, W. E.; Rojas, I.; Azofeifa, D. E.; Clark, N. Thin Solid Films 2006, 496, 189-196.

(26) Weaver, J. H. Phys. Rev. B 1975, 11, 1416-1425.

(27) Weaver, J. H.; Benbow, R. L. Phys. Rev. B 1975, 12, 3509-3510.

(28) Christensen, N. E. Phys. Rev. B 1976, 14, 3446-3461.

(29) Mueller, F. M.; Freeman, A. J.; Dimmock, J. O.; Furdyna, A. M. Phys. Rev. B 1970, 12, 4617-4635.

(30) Fong, C. Y. J. Phys. F: Metal Phys. 1974, 4, 775-782.

(31) Johnson, P. B.; Christy, R. W. Phys. Rev. B 1972, 6, 4370-4379.

(32) Bigall, N. C.; Härtling, T.; Klose, M.; Simon, P.; Eng, L. M.; Eychmüller, A. Nano Lett. 2008, 8, 4588-4592.

(33) Sturm, K.; Schülke, W.; Schmitz, J. R. Phys. Rev. Lett. 1992, 68, 228-231.

(34) Langhammer, C.; Zoric, I.; Kasemo, B.; Clemens, B. M. Nano Lett. 2007, 7, 3122-3127.

NL803794H 